\title{
G-FORCE EXPOSURE IN A CONTACT SPORT: THE USE OF ACCELEROMETERS TO QUANTIFY PHYSICAL STRESS
}

\author{
M.P. McHugh ${ }^{1}$--- S.Y. Kwiecien* --- M.P. Hannon ${ }^{3}$--- R.J. Stewart ${ }^{4}$

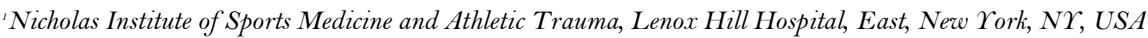 \\ ${ }^{2, s, 4}$ Nicholas Institute of Sports Medicine and Athletic Trauma, Lenox Hill Hospital, East, New York, NY, USA
}

\begin{abstract}
GPS-based analyses of movement patterns are inadequate for quantifying the physical stress imposed on athletes in contact sports. Accelerometers may be useful for monitoring such athletes. The purpose of this study was to use triaxial accelerometers to record G-force exposure in Gaelic football players to examine differences in $G$-force exposure between games and practices and between playing positions. A small triaxial accelerometer with a flash memory chip data logger was fitted to the waistband of players' shorts. Game

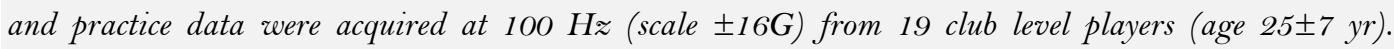
Resultant $G$-force was: calculated for each data point from the raw accelerometer data collected in all three planes of motion and analyzed by quantifying the time spent above different G-force thresholds. Player positions were categorized as central (half backs/half forwards/midfield) and peripheral (full back/full forward) with central players typically more involved in continuous running. ANOVA with Bonferroni corrections was used to compare G-force exposure between games vs. practices, and between central vs. peripheral players (practice not position dependent). Differences between games and practices were apparent for time $>3 G,>4 G$, and $>5 G$ with more time at these thresholds in practices versus games $(P=0.003)$, indicating higher $G$-force exposure in practices versus games. In games central players had greater time at $>2 G$ and $>3 G(P<0.001)$. Accelerometry analysis was effective in distinguishing player position-dependent differences in $G$-force exposure, and differences between games and practices. These differences indicate that the method of analysis of $G$-force exposure used here may be of utility for quantifying the physical stresses imposed on athletes in contact sports.
\end{abstract}

Keywords: Accelerometer, Football, Stress, Playing position, Plane of motion, Movement pattern. 


\section{Contribution/ Originality}

This study is one of very few studies that have investigated physical stresses imposed on athletes in contact sports. Triaxial accelerometers can provide important information regarding the physical stress of an athletic exposure. These data may be important for monitoring playing intensity in a game or accumulated stress over a season.

\section{INTRODUCTION}

Global Positioning System (GPS) sensors are increasingly used to monitor movement patterns in team sports in an attempt to quantify the physical stress placed on the athletes (Cummins, 2013). These sensors give an indication of distance covered and relative velocities. However, in many team sports the primary stress on the athlete comes from accelerations, decelerations and physical contact. This is particularly true in sports such as American football, rugby, Australian rules football and Gaelic football where high stresses are imposed on athletes with sudden directional changes and collisions. GPS sensors are not effective for monitoring these stresses. The analysis of $\mathrm{G}$-force exposure as a measure of physical stress on humans has been studied extensively in pilots in high performance fighter aircraft (Knudson, 1988; Newman and Callister, 1999; Sevilla and Gardner, 2005). Excessive exposure to high G-forces has detrimental physical and physiological effects on pilots (Knudson, 1988; Sevilla and Gardner, 2005). Accelerometers are being used increasingly to document impact forces in contact sports, particularly with regard to head injuries and concussions (Naunheim, 2000; Crisco, 2004; Duma, 2005; Duma and Rowson, 2009; Crisco, 2010; Eckner, 2011; Daniel, 2012; Cobb, 2013; Urban, 2013). Accelerometers can also be used to quantify the whole body physical stress on athletes in contact sports, sports with sudden directional changes (Boyd, 2011; Sullivan, 2013), and differentiating between different types of activity in a single sport (Boyd, 2011). Accelerometers are typically combined with GPS and gyroscopes to identify direction of movement and differentiate accelerations from decelerations. However, these units are expensive (approximately US $\$ 4,000$ each) and not practical for widespread use among all players within a team or across several teams. Triaxial accelerometers without GPS and gyroscope technology are more affordable (less than US $\$ 200$ each) and might be useful in quantifying stresses imposed in athletes in contact sports. However, there has been limited research using accelerometers to quantify whole body physical stresses imposed on athletes in contact sports. The primary purpose of this study is to use triaxial accelerometers to record G-force exposure in Gaelic football players during games and practices. Differences in G-force exposure between games and practices and between playing positions were examined.

\section{MATERIALS AND METHODS}

G-force data was recorded from 19 players (age $25 \pm 7 \mathrm{yr}$, height $1.78 \pm 0.06 \mathrm{~m}$, body mass $80.2 \pm 2.6 \mathrm{~kg}$ ). A small triaxial accelerometer (mass $5 \mathrm{~g}$ ) with a flash memory chip data logger (Axivity, Newcastle, UK) was fitted to the inside center-rear of the waistband of players' shorts. 
Data were acquired at $100 \mathrm{~Hz}$ (scale $\pm 16 \mathrm{G}$ ). Game data were collected from 17 players in seven different games, with two players instrumented in two different games and the rest of the players in a single game (total 19 player-games). Practice data were collected from seven players in four different practice sessions, with two practice sessions recorded for one player and 1 session for the other six players (total 8 player-practice sessions). In Gaelic football there are 15 players from each team on the field at a given time (14 outfield players and 1 goalkeeper). Goalkeepers were not instrumented in this study. Two outfield players are designated as midfielders, whose primary purpose is to catch the ball and distribute it to offensive players. The remaining 12 outfield players are equally divided into forwards (offense) and backs (defense). These players are further subdivided into full backs/forwards and half backs/forwards, with the full backs/forwards playing closer to goal and the half backs/forwards playing more centrally. For the purposes of analysis players were categorized as central (midfielders, half backs/forwards) and peripheral (full backs/forwards). At least one central and one peripheral player were instrumented in each game. The dimensions of the playing field for the games were $140 \mathrm{~m}$ long by $80 \mathrm{~m}$ wide.

In Gaelic football the ball is moved by kicking (primarily kicked from hands) or punching. Proper tackling involves direct shoulder-to-shoulder tackles with deliberate contact to the chest or back penalized. Such contact occurs frequently sometimes deliberately (and penalized) and often inadvertently. A player cannot carry the ball for more than four steps without taking a bounce or tapping the ball from the foot to the hand. Physical contact is common when player attempts to move with the ball in this fashion. Most physical contact occurs when players are attempting to gain possession of the ball (i.e. when neither team has clear possession).

Games consisted of two $30 \mathrm{~min}$ halves with each game preceded by a warm-up of approximately $10 \mathrm{~min}$, and a $10 \mathrm{~min}$ halftime break involving no physical activity. All players that were instrumented played in the full game (i.e. were not substituted). Practices consisted of equal durations of ball work and non-ball work. The ball work involved some physical contact, with no physical contact in the non-ball work. All activities involved running with a predominance of sprinting and/or directional changes (e.g. shuttle sprints).

From the raw accelerometer data collected in all three planes of motion a resultant G-force was calculated for each data point:

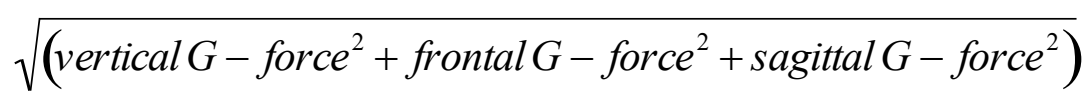

At rest, with the subject standing still, the vertical $\mathrm{G}$-force $=1$ and the horizontal G-forces (frontal and sagittal) $=0$. Resultant G-force data was analyzed by quantifying the time spent above different $\mathrm{G}$-force thresholds, specifically time $>1 \mathrm{G},>2 \mathrm{G},>3 \mathrm{G},>4 \mathrm{G},>5 \mathrm{G},>6 \mathrm{G},>7 \mathrm{G}$, $>8 \mathrm{G}$.

\section{STATISTICAL ANALYSIS}

Time spent at the different G-force thresholds was compared (1) between games and practices, (2) between central and peripheral players using mixed model analysis of variance, with 
repeated measures for time above different G-forces, and game versus practice or central versus peripheral positions as between subjects factors. Where there was a significant interaction between the two factors, differences between games versus practices or between central and peripheral players were assessed by independent t-tests with Bonferroni corrections for planed pairwise comparisons (P value multiplied by 8 for comparisons at 8 different $\mathrm{G}$-force thresholds). Mean and SD are reported in the results and displayed in the figures.

\section{RESULTS}

Games and practices had a similar duration (Game $73 \pm 10 \mathrm{~min}, 10 \mathrm{~min}$ halftime break not included; Practice $75 \pm 5 \mathrm{~min}$ ). The variation in duration of games was due differences in injury time, and differences in warm-up and cool down times between games. The raw accelerations in all three planes for one player in a game are shown in figure 1.

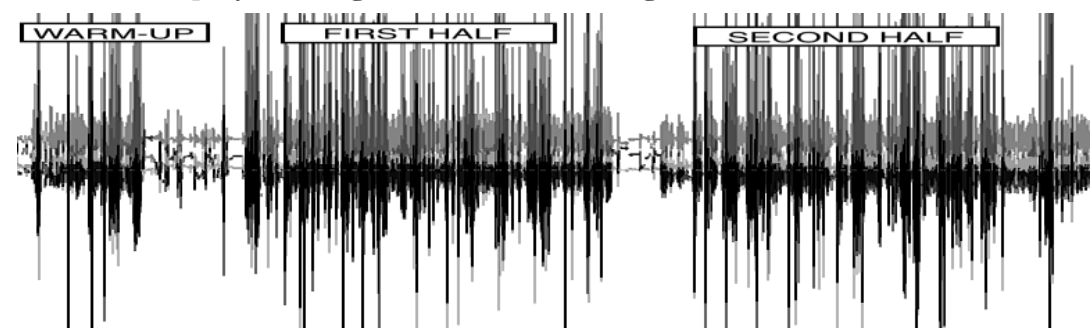

Figure-1. Raw accelerations in all three planes of motion. The first period of activity is the warm-up, followed by the first and second halves, and a cool down period at the end. The vertical plane is separated from the two horizontal planes due to baseline G-force being 1 in the vertical plane and zero in the horizontal planes.

The resultant G-force analysis for games and practices indicated that most activity was less that $2 \mathrm{G}$ with approximately $7-8 \mathrm{~min}$ was spent at $>2 \mathrm{G}$ in games and practices (fig. 2).

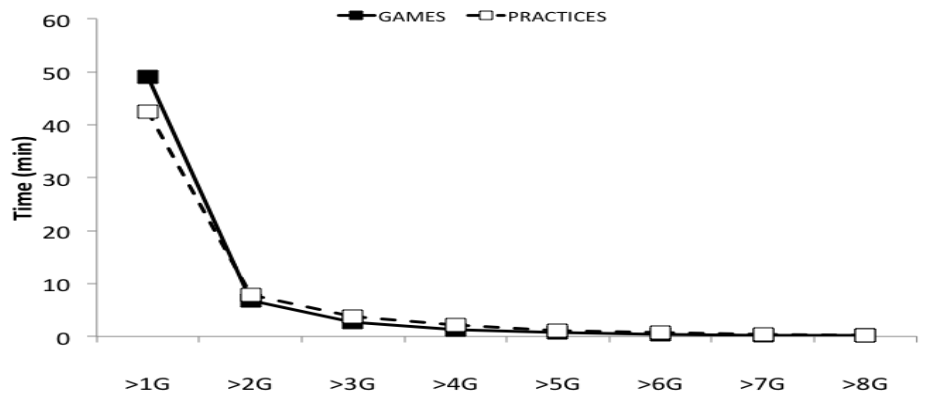

Figure-2. Time at different G-force thresholds showing that most activity is $<2 \mathrm{G}$. Activity $>2 \mathrm{G}$ represents high intensity activity.

Differences in $\mathrm{G}$-force exposure between games and practices were apparent for time $>3 \mathrm{G}$, $>4 \mathrm{G}$, and $>5 \mathrm{G}$ ( $\mathrm{P}=0.003$; fig. 3) with more time at these thresholds in practices versus games, indicating higher G-force exposure in practices versus games. This effect is likely due to the 
emphasis on sprinting and quick directional changes in training (time at $>1 \mathrm{G}$ was not different between games and practices).

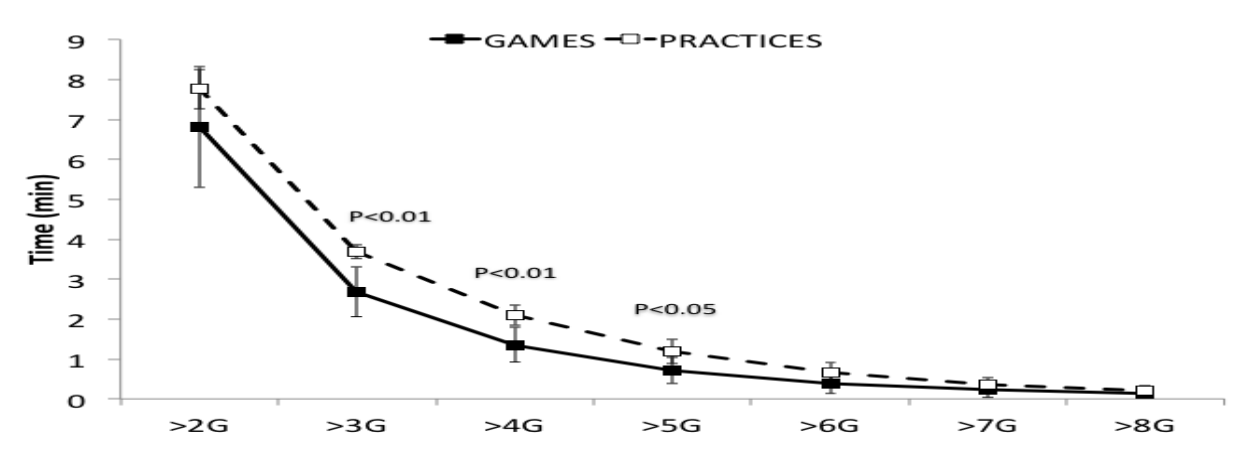

Figure-3. Differences in time at the range of G-force thresholds for games and practices.Games vs. Practices by G-force threshold interaction $P=0.003$. Practices had significantly greater $G$-force exposure at $>3 G,>4 G$ and $>5 G$.

In games central players had greater $\mathrm{G}$-force exposure than peripheral players $(\mathrm{P}<0.001$; Fig. 4 ), with differences most apparent at $>2 \mathrm{G}$ and $>3 \mathrm{G}$. Central players spent $26 \%$ and $27 \%$ more time at $>2 \mathrm{G}$ and $>3 \mathrm{G}$, respectively, than peripheral players. These differences likely reflect the greater running and jumping demands for these players.

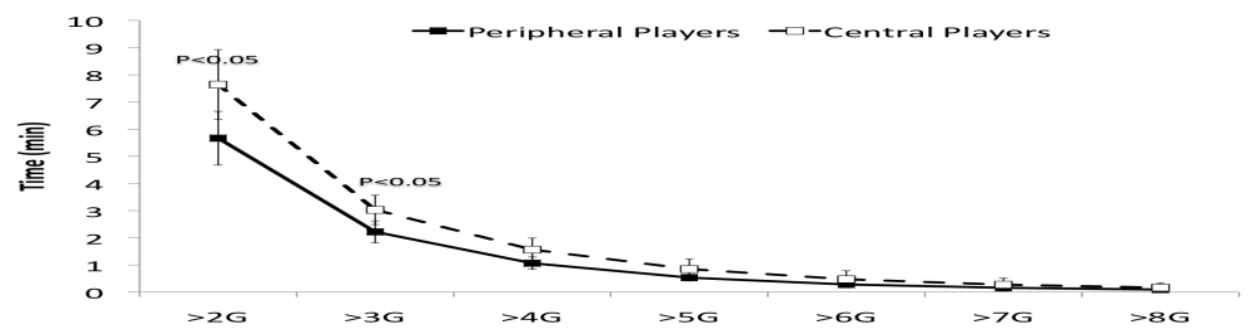

Figure-4. G-force exposure for central versus peripheral players.G-force exposure by player position $\mathrm{P}<0.001$. Greater Gforce exposure for central players at $>2 \mathrm{G}$ and $>3 \mathrm{G}$.

\section{DISCUSSION}

\subsection{Summary of Results}

Accelerometry analysis was effective in distinguishing player position-dependent physical demands in these non-elite club level Gaelic football players. Specifically, players in more central positions (half backs, half forwards, midfielders) had higher G-force exposure than players in more peripheral positions (full backs, full forwards). Additionally, differences were also apparent between games and practices. Specifically, practices had higher G-force exposure than games.

\subsection{Player Position-Dependent Differences in G-Force Exposure}

The greater G-force exposure for central players is likely due to the greater running demands for these players and their more continuous involvement in play. Similar position- 
dependent differences have been reported in elite Australian football using a similar accelerometers device (Boyd et al., 2013). Australian football has many similarities to Gaelic football with an international series of a hybrid of the two games played semi-annually. Therefore, comparison of accelerometer data between Australian and Gaelic football is practically relevant. However, a direct comparison of G-forces is not possible as the device used in Australian football reported "player load" in arbitrary units. Additionally the accelerometer had a range of $\pm 6 \mathrm{G}$, whereas the unit used here had a range of $\pm 16 \mathrm{G}$, with the data reported in $\mathrm{G}$-force rather than arbitrary units. In Australian football midfielders and nomadic players have greater running demands and more continuous involvement in play than deep forwards and defenders. Thus this represents a similar dichotomy to the central versus peripheral dichotomy used in the present study to analyze position-dependent differences in G-force exposure in Gaelic football players. In Australian football player load for peripheral players (deep forwards and defenders) was $71-74 \%$ of the load for central players (midfielders and nomadic players). In the present study time at $>2 \mathrm{G}$ for peripheral players was $74 \%$ of that for central players. Similarly, time at $>3 \mathrm{G}$ for peripheral players was $73 \%$ of that for central players (Fig. 4). These differences are remarkably similar to the position-dependent differences in player load reported for Australian football.

It is worth noting that in recent years playing strategy in Gaelic football at the highest level has changed in that full backs have begun to get more involved in the play and no longer confine themselves to defending immediately in front of goal. This change in strategy may be in recognition of the fact that they were being underutilized with respect to capacity for physical exertion. It would be interesting to see if G-force exposure in such players was similar to that of the centrally located players. The team in this study did not adopt such tactics.

\subsection{Differences in G-Force Exposure between Games and Practices}

It may seem paradoxical that $G$-force exposure was higher in practices versus games. However, there are several reasons for this. Firstly, practices involved repeated running drills (with and without the ball) at high speed and with numerous directional changes. Secondly, game practices involved approximately 7 -a-side games, which are typically played at a higher tempo than regular games. Thirdly, player positions are not relevant in practices as all outfield players do the same drills and small-sided practice games do not involve the typical player positions. Of note, Boyd et al. (2013) reported similar or higher player loads in small-sided games in practices compared with actual games in Australian football. The greater G-force exposure in practices versus games in the present study is in agreement with the results of Boyd et al. (2013).

Typically in Gaelic football there is more physical contact in games versus practice. However, in the present study G-force exposure was significantly higher in practices versus games in terms of time spent at $>3 \mathrm{G}$ ( $37 \%$ higher), $>4 \mathrm{G}$ ( $54 \%$ higher) and $>5 \mathrm{G}$ (66\% higher). Since time spent at $>9 \mathrm{G}$ amounted to less than $15 \mathrm{~s}$ for all players in either games or practices, the primary analysis did not include stepwise analysis of G-force thresholds for these high forces. However, it is likely that a lot of physical contact occurs at such high G-forces. It is worth noting that time at $>13 \mathrm{G}$, 
$>14 \mathrm{G},>15 \mathrm{G}$ and $>16 \mathrm{G}$ was $30-38 \%$ higher in games versus practices, but the differences were not statistically significant. Average time at $>13 \mathrm{G}$ was $687 \mathrm{~ms}$, diminishing to $230 \mathrm{~ms}$ for time spent at $>15 \mathrm{G}$ and $126 \mathrm{~ms}$ for time spent at $>16 \mathrm{G}$. An example of an illegal hit sustained by a player in one of the games resulting in a caution is shown in Figure 5. The player was hit in the chest while jumping to catch a ball and was subsequently diagnosed with severely bruised ribs.

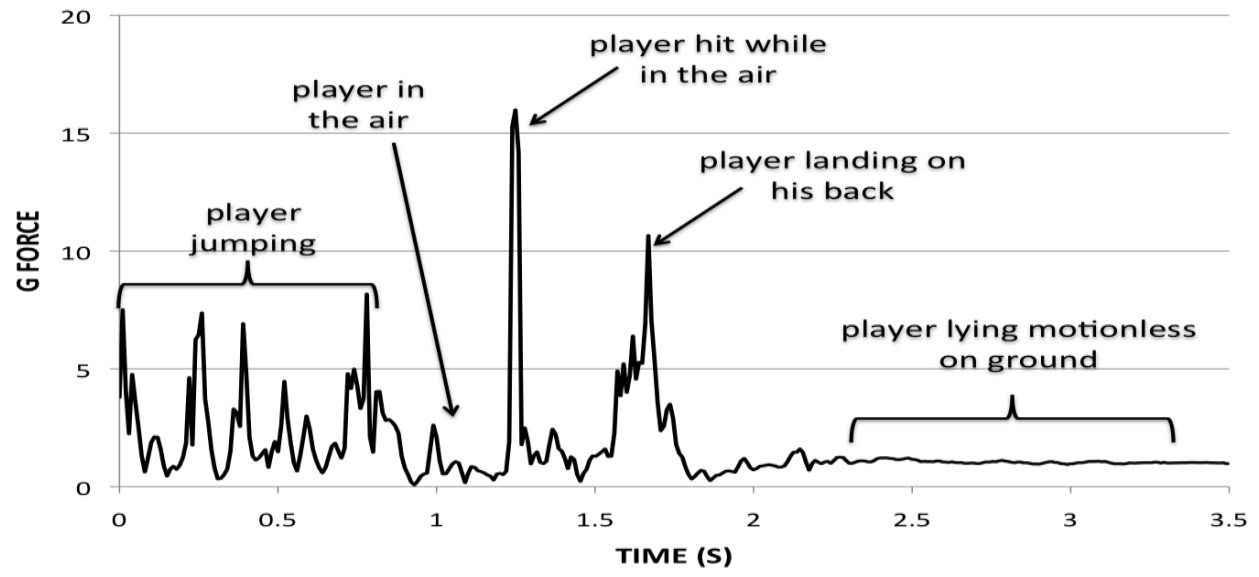

Figure-5. Example of an illegal hit where a player was struck in his chest by the shoulder of another player while catching the ball. The G-force approximates zero while the player was in the air until the hit occurred (approximately $16 \mathrm{G}$ hit). The next large deflection in $G$-force is the player landing on the ground after the hit. Finally the $G$-force plateaus at $1 \mathrm{G}$ as the player lay motionless on the ground.

\subsection{Limitations}

Since this was a pilot study to examine the utility of these accelerometers for assessing physical stress in contact sport athletes only a small number of units were purchased. Thus it was not possible to instrument all players in all games. The coaches sought to have as many different players instrumented and chose specific players for specific games, with instruction to chose at least one central and one peripheral player per game. Four players were instrumented in both games and practices. For these players time at $>3 \mathrm{G},>4 \mathrm{G}$ and $>5 \mathrm{G}$ for games was $64 \%, 59 \%$, and $59 \%$ of the values for practices. These differences were very similar to the values for the whole sample of players $(73 \%, 65 \%, 60 \%)$. Thus the differences between games and practices reflected the true differences in G-force exposure as opposed to differences in G-force exposure between different players monitored in games versus practices.

It was not within the scope of this study to examine the variability in G-force exposure within players across multiple games. However, one player was instrumented for 2 different games and one player was instrumented for 2 different practices. The measurements were very similar between the first and second game and practices, respectively (fig. 6). 


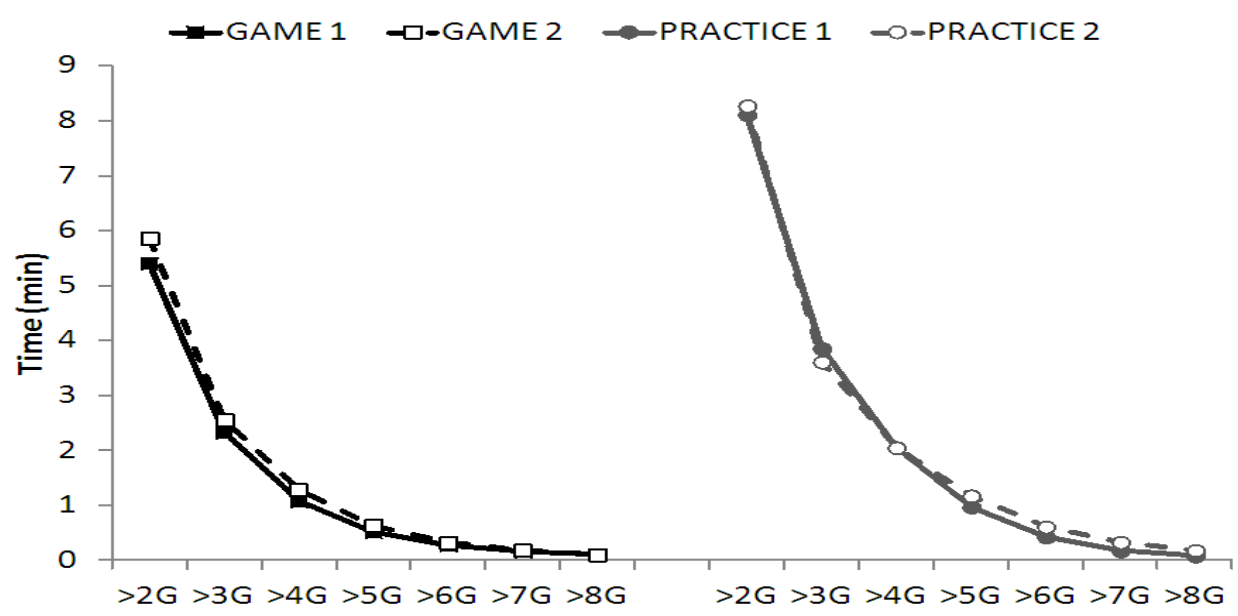

Figure-6. Measurement variability for G-force exposure for one player playing in two different games, and a different player in two different practices. G-forces were very consistent between athletic exposures.

Since the accelerometer used in this study was not combined with GPS and a gyroscope, it was not possible to accurately differentiate between accelerations in the sagittal, frontal and vertical planes, nor was it possible to differentiate between accelerations and decelerations. Analysis of decelerations may be important for analyzing physical contact and hits. Additionally decelerations may be more relevant to stress on the musculoskeletal system since they will necessarily involve eccentric muscle contractions.

\subsection{Conclusions and Practical Relevance}

The popularity of GPS derived, or video derived, analysis of distances covered in team sports such as soccer, rugby and Australian football has been increasing. However, such data cannot truly represent the stress imposed on athletes in such sports as much of the stress occurs with accelerations and decelerations, including physical contact. For example, the typical distance covered in a 90 min soccer game is $10 \mathrm{~km}$ (Randers, 2010) while in an 80 min Australian football game total distance is in the region of $12 \mathrm{~km}$ (Gray and Jenkins, 2010). This is the equivalent of $6.6 \mathrm{~km} / \mathrm{hr}$ and $9.0 \mathrm{~km} / \mathrm{hr}$, respectively, neither of which represents a significant physiological stress for moderately fit athletes. Clearly the major stress is not coming from the distances covered rather the efforts in accelerations, decelerations, directional changes and collisions. Hence the relevance of accelerometer-based analysis of player movements.

The player position-dependent differences in G-force exposure, and differences between games and practices, indicate that the method of analysis of G-force exposure used here may be of utility for quantifying the physical stresses imposed on athletes in Gaelic football. The fact that the position dependent differences, and differences between games and practices, are similar to those observed in Australian football using a different accelerometer and a different method of 
analysis, indicates that this method of quantifying G-force exposure may be relevant to other sports involving high speed running, with multiple directional changes and physical contact. With respect to the analysis of G-force exposure in Gaelic football, time at $>2 \mathrm{G}$ and time at $>3 \mathrm{G}$ may be good measures of physical stress imposed on athletes, and may provide a measure of the intensity of a given athletic exposure.

Funding: This study received no specific financial support.

Competing Interests: The authors declare that they have no competing interests.

Contributors/Acknowledgement: All authors contributed equally to the conception and design of the study.

\section{REFERENCES}

Boyd, L.J., 2011. The reliability of minimaxX accelerometers for measuring physical activity in Australian football. Int J Sports Physiol Perform, 6(3): $311-321$.

Boyd, L.J., 2011. A new way of using accelerometers in Australian rules football: Assessing external loads. Doctoral Dissertation, Victoria University.

Boyd, L.J., K. Ball and R.J. Aughey, 2013. Quantifying external load in Australian football matches and training using accelerometers. Int J Sports Physiol Perform, 8(1): 44-51.

Cobb, B.R., 2013. Head impact exposure in youth football: Elementary school ages 9-12 years and the effect of practice structure. Ann Biomed Eng., 41(12): 2463-2473.

Crisco, J.J., 2004. An algorithm for estimating acceleration magnitude and impact location using multiple nonorthogonal single-axis accelerometers. J Biomech Eng, 126(6): 849-854.

Crisco, J.J., 2010. Frequency and location of head impact exposures in individual collegiate football players. J Athl Train, 45(6): 549-559.

Cummins, C., 2013. Global positioning systems (GPS) and microtechnology sensors in team sports: A systematic review. Sports Med, 43(10): 1025-1042.

Daniel, R.W., 2012. Head impact exposure in youth football. Ann Biomed Eng, 40: 976-981.

Duma, S.M., 2005. Analysis of real-time head accelerations in collegiate football players. Clin J Sport Med, 15: 3-8.

Duma, S.M. and S. Rowson, 2009. Every newton hertz: A macro to micro approach to investigating brain injury. Conf Proc IEEE Eng Med Biol Soc. pp: 1123-1 126.

Eckner, J.T., 2011. No evidence for a cumulative impact effect on concussion injury threshold. J Neurotrauma, 28: 2079-2090.

Gray, A.J. and D.G. Jenkins, 2010. Match analysis and the physiological demands of australian football. Sports Med., 40(4): 347-360. [Accessed 2010 Apr 1].

Knudson, R., 1988. A comparative study of G-induced neck injury in pilots of the F/A-18, A-7, and A-4. Aviat Space Environ Med, 59: 758-760.

Naunheim, R.S., 2000. Comparison of impact data in hockey, football, and soccer. J Trauma, 48: 938-941.

Newman, D.G. and R. Callister, 1999. Analysis of the Gz environment during air combat maneuvering in the F/A-18 fighter aircraft. Aviat Space Environ Med, 70: $310-315$. 
Randers, M.B., 2010. Application of four different football match analysis systems: A comparative study. J Sports Sci, 28: 171-182.

Sevilla, N.L. and J.W. Gardner, 2005. G-induced loss of consciousness: Case-control study of 78 G-Locs in the F-15, F-16, and A-10. Aviat Space Environ Med, 76: 370-374.

Sullivan, C., 2013. Match score affects activity profile and skill performance in professional Australian football players. J Sci Med Sport, 17: 326-331.

Urban, J.E., 2013. Head impact exposure in youth football: High school ages 14 to 18 years and cumulative impact analysis. Ann Biomed Eng, 41: 2474-2487. 